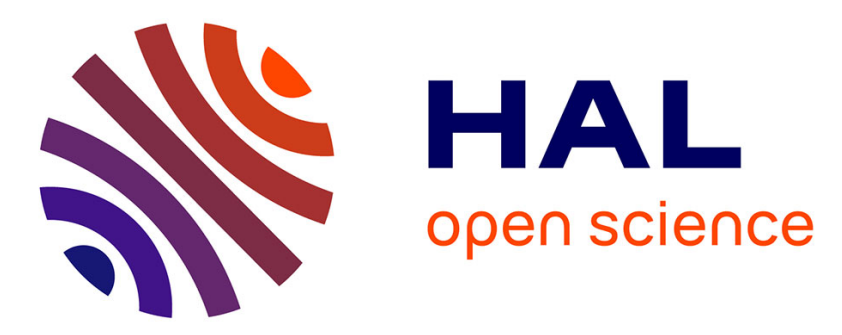

\title{
Strategic Planning of Aircraft Trajectories in North Atlantic Oceanic Airspace Based on Flocking Behaviour
}

Imen Dhief, Nour Elhouda Dougui, Daniel Delahaye, Noureddine Hamdi

\section{To cite this version:}

Imen Dhief, Nour Elhouda Dougui, Daniel Delahaye, Noureddine Hamdi. Strategic Planning of Aircraft Trajectories in North Atlantic Oceanic Airspace Based on Flocking Behaviour. 2016. hal01302651

\section{HAL Id: hal-01302651 \\ https://hal-enac.archives-ouvertes.fr/hal-01302651}

Preprint submitted on 29 Jun 2016

HAL is a multi-disciplinary open access archive for the deposit and dissemination of scientific research documents, whether they are published or not. The documents may come from teaching and research institutions in France or abroad, or from public or private research centers.
L'archive ouverte pluridisciplinaire HAL, est destinée au dépôt et à la diffusion de documents scientifiques de niveau recherche, publiés ou non, émanant des établissements d'enseignement et de recherche français ou étrangers, des laboratoires publics ou privés. 


\title{
Strategic Planning of Aircraft Trajectories in North Atlantic Oceanic Airspace Based on Flocking Behaviour
}

\author{
Imen Dhief* ${ }^{\dagger}$, Nour Houda Dougui*, Daniel Delahaye ${ }^{\dagger}$ and Noureddine Hamdi* \\ *Heterogeneous Advanced Networking and Applications (HANA) Research group ENSI \\ Manouba University Campus, 2010 Manouba, Tunisia \\ ${ }^{\dagger}$ Laboratory of Applied Mathematics, Informatics and Automatics for Aviation (MAIAA) ENAC \\ 7 avenue Edouard Belin, 31055, Toulouse, France
}

\begin{abstract}
The North Atlantic Airspace (NAT) accommodates traffic between Europe and North America. This area is considered as the most congested oceanic airspace in the world. Radar-Based surveillance is not applied in the most of the oceanic area due to its limited coverage. So, aircraft become obliged to follow predefined routes called Organized Track System (OTS). These routes require very restrictive separation standards which limit the traffic of aircraft. Thus, a new kind of communication system, called Automated Dependence Surveillance Broadcast (ADS-B), has been introduced in order to afford the aircraft a reliable communication with both controllers and surrounding traffic. Hence, aircraft crossing the NAT will be able to follow more flexible routes, which will improve significantly the air traffic situation over this area. In this paper, we propose a strategic planning ${ }^{1}$ model that overcomes the constraints of the OTS system in order to produce the closest routes to the direct ones of aircraft. This method is based on flocking boid model. It provides us with satisfying results on a portion of one day traffic over the NAT airspace.
\end{abstract}

\section{INTRODUCTION}

It is worth-noting that the air navigation in the oceanic area differs from the continental one because of the lack of the navigational and communication equipments. Thus, aircraft are required to follow predefined routes and respect highly restrictive separation standards. The Organized Track System (OTS) is a route structure that has been established in the North Atlantic Oceanic area. These routes have been constructed in order to handle as many flights as possible on, or close to, their minimum time tracks and altitude profiles [1]. The new Automated Dependance Surveillance Broadcast (ADS-B) technology is an airborne-based system that provides both controllers and pilots with more accurate and reliable information. In fact, the aircraft transponders receive GPS signals to define the aircraft actual position. Then, the position as well as other information are broadcast and sent to both

\footnotetext{
${ }^{1}$ Strategic planning: this step consists in constructing a day traffic in order to overcome the constraints of the sector capacities with better considering the needs of the airlines companies. This planning is done before the plane taking-off.
}

the aircraft neighbours and the controllers. Due to ADS$B$, the air traffic situation in the oceanic air space has been improved. Thus, flights are allowed to follow more flexible and direct routes from the departure airport to the arrival one. In this study, we propose a strategic planning of aircraft trajectories while transiting flights off the predefined structure routes OTS.

This planning method is inspired from the Flocking swarm model named "Boids Flocking", which is a swarm model introduced by Craig Reynold's in the 1980s [2]. Reynold's implemented an algorithm for simulating animal motion such as bird flocks and fish school, where boids refer to the generic flocking simulated creature. The aggregate motion represents the result of interaction between the simple behaviour of each boid.

Recently, the paradigm of multi-agent systems has become widely applied for air traffic management. Several researches are devoted to apply multi-agent systems for air traffic management and collision avoidance in the continental airspace [3, 4]. Nevertheless, to our knowledge, no research works has applied these systems in the oceanic airspace. This paper introduces an algorithm based on flocking model for strategic planning aircraft over the NAT airspace. We suppose that all aircraft are equipped with ADS-B system. We take advantages from the reliable information that can be transmitted between flights to construct a full swarm behavior. Thus, each aircraft is considered as an agent. Besides, all agents communicate with each other. They adjust their caps in order to avoid conflict while maintaining the swarm behavior.

The rest of this paper is organized as follows : The next section presents a brief description of the North Atlantic airspace and defines the principle of the Flocking model. Section III contains relevant works on optimum trajectory generation in the oceanic area. Section IV exposes the problem formulation, and shows how we adapt the Flocking model to construct conflict-free trajectories in North Atlantic airspace. The simulation and computational results are presented and analyzed in section V. Finally, section VI presents some concluding remarks. 


\section{BACKGROUND}

In this part, we define the main concepts related to our research subject. First, we describe the North Atlantic airspace. Then, we display the Flocking model which is the key element of our approach.

\section{A. North Atlantic Airspace (NAT)}

The Atlantic Oceanic covers a total area of about $106,400,000$ square kilometers. It is considered as the second largest oceanic airspace after the Pacific airspace. North Atlantic Airspace accommodates traffics between Europe and North America. Daily, about 1000 transatlantic flight cross this area in each direction, which makes it the busiest oceanic airspace.

Since radar coverage is limited in the middle of the Atlantic airspace, aircraft are required to follow predefined tracks referred to as the Organized Track System (OTS). Besides, they are compelled to respect highly restrictive separation standards. The organized track system is constructed to be as closer as possible to the minimum time and to the altitude profiles of flights. NAT traffic was divided into two major flows: a westbound flow from Europe to North America in the morning and an eastbound flow from North America to Europe in the evening. Thus, a congestion of this region is detected in the west direction between $11 \mathrm{~h} 30$ and $18 \mathrm{~h} 00 \mathrm{UTC}$ and in east direction between $01 \mathrm{~h} 00$ and 08h00 UTC.

The OTS includes between 5 to 7 tracks in each direction for different flight level ${ }^{2}$. In order to ensure safety, these tracks are separated by 60NM laterally and 1,000 feet vertically. Around 10 waypoints are planned in each track. Transatlantic flights using OTS tracks are required to follow the same track, and any rerouting decision should be taken in the waypoints. The longitudinal separation standards, which is defined between two consecutive aircraft following the same track, is defined by 10 minutes if aircraft are following the same track and by $15 \mathrm{~min}$ utes when an aircraft is changing its track. These very demanding separation standards result from the lack of surveillance coverage in the oceanic area. As the traffic in this region increases, these procedural separations generate inefficiencies in oceanic operations and limit oceanic air routes capacities. Considering these issues, an innovative technology, known as Automatic Dependence SurveillanceBroadcast (ADS-B), has been recently introduced by the International Civil Aviation Organization (ICAO). The ADS-B systems rely upon automatic position reports by making use of satellites to provide global coverage. ADS$\mathrm{B}$ is a surveillance application that transmits periodically aircraft information (position, velocity and heading, etc...) via a broadcast mode data link to both controllers and surrounding traffics. Thanks to the accuracy of this communication tools, a significant reduction in separation standards can be accommodated. In fact, the longitudinal

\footnotetext{
${ }^{2}$ Flight level is a pressure altitude expressed in hundreds of feet.
}

separation between aircraft becomes 2 minutes if aircraft are successive in the same track, and 3 minutes if an aircraft changes its track. Further details about the functionalities and benefits behind the design and development of this advanced technology are given in [5] and [6].

\section{B. Flocking model}

In nature, flocks could be considered as self-organized networks of mobile agents that cooperate with each other to form group behaviour. The flocking model was introduced by Craig Reynold's in 1986 [2]. It represents the form of collective behavior of a large number of interacting agents with a common group objective. It is especially exploited to provide realistic life-look representations of the aggregate motion of animal groups, such as birds, fish and sheep. In Reynold's model, each agent was called "boid". The boid model has the three following heuristic rules applied to the boids at each time sample:

- Separation (figure 1 (a)) : this rule is applied in order to maintain a specific separation distance between agents. Each boid is required to ensure a separation distance with his flock mates to avoid collision and prevent crowding.

- Alignment (figure 1 (b)): is performed in order to oblige boids to follow each other in quasi-parallel trajectories. Each boid matches the direction and the speed of its neighbors.

- Cohesion (figure 1 (c)): this rule is performed in order to ensure the swarm behaviour. Each boid moves to the average position of its neighbours.



(a) Separation rule

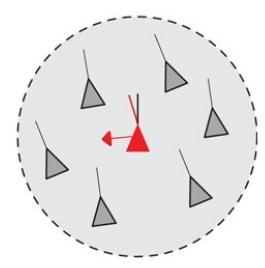

(b) Alignment rule

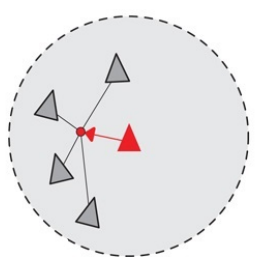

(c) Cohesion rule
Fig. 1: The boids model rules

It is important to note that the separation rule acts as a complement of the cohesion rule. In fact, if only cohesion is applied all boids will merge into the same point. Figure 1 illustrates the behavior of an agent when applying the three flocking forces.

\section{RELATED WORK}

There are several works aiming to improve air traffic situation in the oceanic airspace. The first study, that examined the benefits of transitioning from the fixed predefined routes to user-preferred routes in the Central East Pacific (CEP) airspace, was proposed in[7]. In this survey the user's preferences were assumed to be 
Wind-optimal. The simulation of new aircraft trajectories was developed via a minimum-travel-time and Windoptimal dynamic programming(DP). The potential time and distance gain were $9.9 \mathrm{~min}$ and $36 \mathrm{NM}$ per flight, respectively. This research proved that significant benefits could be achieved by surrogating the fixed routes to use wind-optimal routes. After constructing the new windoptimal trajectories structure, the same authors treated the problem of conflict detection and its resolution in[8]. The problem was modeled as a job shop scheduling issue and was solved afterwards via 0-1 integer programming model. The maneuvering resolution consists in calculating the optimal departure and en-route delays. This method was tested with a set of 175 flights and with two different sizes of the machine in the job shop. The first type, where the size of machine was $30 \mathrm{NM}^{*} 30 \mathrm{NM}$, generates a conflictfree trajectories. However, it is highly restrictive since it enhances the computational complexity of the problem by increasing the number of variables and constraints. The second algorithm, where the size of machine was $100 \mathrm{NM}^{*} 100 \mathrm{NM}$, is less restrictive, but it may require further tactical separation maneuvers. It was thought that this method achieved considerable economic benefits to the airlines when applied on the CEP. Nevertheless, in this paper, we solve the problem of air traffic in the North Atlantic Airspace which accommodates daily about 1,000 flights in each direction. To deal with this issue, we cannot apply the above- mentioned method because it may increase exponentially the constraints of the problem, which makes it unsuitable in our study case.

Recent approach of optimizing air traffic over the NAT airspace has been proposed in [9]. In this work, the authors have presented an optimization model to improve the air traffic situation over the OTS tracks when implementing the ADS-B technology. The problem consists in searching for the optimal flight path within the OTS in order to reduce the congestion while exiting the continental airspace.The simulated annealing algorithm has been applied to solve the high complexity of the combinatorial optimization. Computational results prove that operating the ADS-B systems has widely ameliorated the traffic situation. In [10], they used wind-optimal trajectory instead of the north Atlantic Tracks. Therefore, flight trajectories were generated based on wind-optimal routes. Small adjustments in departure time and rerouting have reduced the number of potential conflicts. The deconfliction strategy was ensured with a simulated annealing combined with a local gradient search. The findings show the efficiency of this methodology mainly in fuel saving. In our study, we intend to apply an innovative bio-inspired method based on Multi-Agent algorithm for generating and de-conflicting aircraft trajectories without using the OTS structure. The following section presents the flocking model on which our method is based.

\section{Problem Formulation}

In this section, we start by modeling the flight, subject of our study. Then, we introduce some assumptions in order to simplify the problem. Finally, we explain how we adapt the above-mentioned Flocking model.

\section{A. Flight model}

Each flight is defined by a number of parameter values. Some of these values are fixed data, while others can be modified and represent our scope for reducing conflict. In this paper, we assume that the flight level and the Mach number are constant since we are tackling only the enroute phase ${ }^{3}$. For each flight the following input data are given :

- Entry and exit points in the NAT space are characterized by its latitude and longitude coordinates.

- Cruise altitude in feet which is supposed to be constant for the entire trajectory.

- Desired entry time

- True airspeed in knots is supposed to be constant during the entire trajectory.

Preliminary experiments have proved that this state space is insufficient to guarantee conflict-free trajectories. For this reason, entry delay less than 20 minutes is allowed. Besides, the positions of entry and exit points are not fixed, they can be changed. In fact, if we consider that the desired exit point is the center of a circle having a 5 NM radius, any point of its surface is an potential exit point. This radius may not be sufficient to choose the entry point. In fact, we intend to separate aircraft when they enter at the same point (or when distance between their entry points is less than the separation norm), and the difference between their entry times is less than 3 minutes. The decision variables in our model are given below:

- $\left(p_{i}, \vec{V}_{i}\right), i=1,2,3, \ldots, N$ where $p_{i}\left(x_{i}, y_{i}, z_{i}\right)$ is a sequence of points defining the position of the aircraft at time sample and $\vec{V}_{i}$ is the motion vector applied at the point $p_{i}$.

- $d_{\text {In }}$ the time delay at the entry point

- $P_{I n}$ the assigned entry point

- $P_{\text {Out }}$ the assigned exit point

These variables must be determined to guarantee a set of conflict-free trajectories while respecting the limit of the permitted-delay and the allowed deviation from the desired entry and exit points.

\section{B. Problem model}

This section presents a description of the mathematical model used in our trajectory-planning methodology. In this paper, we intend to construct flight trajectories while adopting the swarm behavior introduced by Reynolds. Since we assume that the flights altitude profile does not

\footnotetext{
${ }^{3}$ The en-route phase of the flight is defined as the segment of flight from the arrival at the initial cruise altitude to the starting of the descent.
} 
change for their entire trajectory, we can work in 2D airspace. We consider the airspace as a Euclidean space. Latitudes and Longitudes in the earth space are projected in $2 \mathrm{D}$ space by a Lambert azimuthal projection with the center of projection is located at the center of the North Atlantic airspace. The flight trajectory is represented as a set of discrete points from the departure to the destination. The range between each two points represents 1 minute navigation with the given data. Initially, we suppose that aircraft cap is directly steered to the destination point. Thereafter, the cap is adjusted in each point in order to avoid conflict with neighbours while maintaining the swarm behaviour.

1) Conflict detection: As we have already mentioned, the separation standards in the oceanic area are greater than the continental ones due to the lack of reliable means of surveillance. Aircraft operating over the NAT airspace are required to maintain lateral, vertical and longitudinal separations. The lateral separation is set to be $60 \mathrm{NM}$ that is equal to approximately $111.11 \mathrm{~km}$, or $1^{\circ}$ of latitude. The minimum vertical separation is 1,000 feet. It is ensured by using different flight level. Using the OTS tracks, these two separation standards are already respected. Thus, the only separation to respect in the OTS is the longitudinal one which is set to be 10 minutes, and it is defined between two consecutive aircraft in the same tracks. In our work, we do not use the OTS tracks. Aircraft are allowed to follow nearly its direct route from its entry point to its destination. Since we assume that all aircraft are equipped with ADS-B system, it is possible to reduce the separation standards. Thus, we consider, in this paper, the lateral separation which is reduced to $20 \mathrm{NM}$ and the current vertical separation which is 1,000 feet between flight levels. Thus, for each aircraft, we can define a protection zone represented as a cylinder with radius equal to the lateral separation $\left(R_{h}=20 N M\right)$, and half-altitude equal to the vertical separation norm $\left(R_{v}=1000\right.$ feet $)$ as shown in figure 2. Thus, a conflict is detected when the protection zone of an aircraft is crossed by another aircraft. In this



Fig. 2: The protection zone

paper, trajectories are sampled with a time step of $\Delta t=60$ second. This time step is small enough to ensure that all potential conflicts are identified. Thus, a flight trajectory is a set of points $\left(p_{i}^{f}, t_{i}^{f}\right)$ where $p_{i}^{f}$ is the position of the concerned flight at the time $t_{i}^{f}=n * \Delta t$ (where $n$ is a positive integer). If we consider two flights $\mathrm{f}$ and $\mathrm{g}$ and their trajectories which are respectively $\left(p_{i}^{f}, t_{i}^{f}\right)$ and $\left(p_{i}^{g}, t_{i}^{g}\right)$. A conflict is detected between $\mathrm{f}$ and $\mathrm{g}$ if at the time $t=t_{i}^{f}=t_{j}^{g}$ the positions $p_{i}^{f}$ and $p_{j}^{g}$ violate both lateral and vertical separation norms. Therefore, conflict at point $p_{i}^{f}$, denoted $\Phi_{i}^{f}$, is defined as the total number of aircraft whose protection zone crosses the protection zone around $p_{i}^{f}$. The conflict associated with the trajectory of the flight $\mathrm{f}$, denoted $\Phi_{f}$ is set as follows:

$$
\Phi_{f}=\sum_{i=1}^{N} \Phi_{i}^{f}
$$

where $N$ is the number of points in the trajectory of the flight $f$. The total number of conflicts between trajectories of the set of flights, $\Phi_{t o t}$, is defined as:

$$
\Phi_{t o t}=\sum_{f \in S} \Phi_{f}
$$

Since aircraft do not have the same speed, another type of conflict can be detected. It happens when an aircraft is slower than the one following it. This conflict must be resolved from the start otherwise our algorithm of resolution can not deal with it. We consider two flights $\mathrm{f}$ and $\mathrm{g}$ and respectively their entry points $\left(P_{i n}^{f}, P_{i n}^{g}\right)$ and exit points $\left(P_{\text {out }}^{f}, P_{\text {out }}^{g}\right)$. Besides, $t_{\text {in }}^{f}$ and $t_{\text {in }}^{g}$ are respectively the entry time of $\mathrm{f}$ and $\mathrm{g}$. If we consider that the two flights $f$ and $g$ satisfy the inequalities in (3), then g may catch up f.

$$
\left\{\begin{array}{c}
\operatorname{distance}\left(P_{i n}^{f}, P_{i n}^{g}\right)<20 N M \\
\operatorname{distance}\left(P_{\text {out }}^{f}, P_{\text {out }}^{g}\right)<20 N M \\
\operatorname{Speed}(f)<\operatorname{Speed}(g) \\
t_{\text {in }}^{f}<t_{\text {in }}^{g}
\end{array}\right.
$$

where the function distance calculates the distance between two points in the space, and Speed gives the velocity of the aircraft. First, we have to calculate the distance crossed by $\mathrm{f}$ at $t_{i n}^{g}$. Then, we compute the time necessary for $g$ to catch up $\mathrm{f}$. If that time is less than the time necessary for $\mathrm{f}$ to reach its destination, then a conflict must occur between $\mathrm{f}$ and $\mathrm{g}$.

2) Defining neighbourhood: For each flight, we define a set of flight neighbours within a predefined distance. In our study, the neighbourhood regions differ according to the application of the flocking rules. As it is illustrated in the figure 3 , the separation area can be defined as the space surrounding the aircraft (red color). In this area, we apply the separation force with the closest aircraft in order to prevent collision. We fix experimentally the radius of this area to be the double of the standard lateral separation between aircraft. The green color in figure 3 shows the area where we apply the alignment force, while the blue area corresponds to the region where we apply the cohesion force to the farther aircraft to make them stay in the group. The alignment and cohesion area are 




Fig. 3: Neighbourhood area

also experimentally tuned to be respectively five and eight times the standard lateral separation.

3) Resolution algorithm: First of all, we sort the flights according to their entry time. Then, we have to ensure that the set of flights is free of conflict in the departure point. Otherwise, we decongest the flights by a sequential method of shifting entry time. In fact, the delay in the entry time of each aircraft must not exceed 20 minutes. If a conflict-free situation at the departure points is not possible with constrained time shifts, it may be necessary to slightly modify the positions of the departure points. The distance between the assigned and the desired entry points must be less than $20 \mathrm{NM}$. Once all the departure conflicts are resolved, we start with the first point of each flight path, beginning from the earliest flight in the set. Then, we calculate the motion vector $\vec{V}_{i}$ to construct the next point in the trajectory. Thus, the motion vector is defined as in (4):

$$
\vec{V}_{i}=60 * V * \frac{\vec{U}_{i}}{\left\|\overrightarrow{U_{i}}\right\|}
$$

where $i$ refers to the aircraft index, $V$ is the speed of the aircraft expressed in meter per second. To have the distance covered in 1 minute navigation, We multiply by 60 seconds. The vector $\vec{U}_{i}$ corresponds to the weighted sum of the five following vectors.

a) The alignment vector of the Flocking model: it causes a flight to line up with its neighbours. Each flight has to adjust its heading. The new heading of each flight is equal to the average of headings of its neighbours. This vector is computed at each point of the flight trajectory as the average of the velocity vector of its neighbours. Thus, the alignment vector is calculated as the displacement vector in (5).

$$
\overrightarrow{A_{i}}=\frac{\sum_{k \in N}\left(\vec{V}_{k}\right)}{N}
$$

where $\vec{A}_{i}$ is the alignment vector of the flight $i, N$ is the number of neighbours of the considered flight $i$ and $\vec{V}_{k}$ is the velocity vector of the neighbours' aircraft $k$. As it is illustrated in the former section, the neighbours flights, considered to compute this vector, are those detected in the green region of figure 3 .

b) The separation vector of the Flocking model: prevents flights from local crowding. To implement this rule, we calculate the separation vector of each flight i $\overrightarrow{S_{i}}$ by applying (6).

$$
\overrightarrow{S_{i}}=\frac{\sum_{k \in N}\left(\left(\vec{P}_{i}-\vec{P}_{k}\right) / \operatorname{dist}^{2}\left(\vec{P}_{i}, \vec{P}_{k}\right)\right)}{N}
$$

where $\vec{S}_{i}$ is the separation vector of the flight $i, N$ is the number of neighbours, $\vec{P}_{i}$ is the position of flight $i$, $\overrightarrow{P_{k}}$ is the position of its neighbours aircraft $k$, and $\operatorname{dist}()$ calculates the distance between two points. In this case, we apply this force to the closest neighboring flights. They are located in the red region of the figure 3 . If only the separation rule is applied, the flights will dissipate.

c) The cohesion vector of the Flocking model: this vector tries to gather the flights to form a swarm behavior. Cohesion of flight $\mathrm{i}$ is calculated by following two steps. First, we compute the center of the neighbours positions of a flight $i$, which we called $\overrightarrow{F C}_{i}$, as in (7). Then, we need to steer the flights toward its neighbours center density by calculating the cohesion displacement vector in (8).

$$
\begin{gathered}
\overrightarrow{F c}_{i}=\frac{\sum_{k \in N} \vec{P}_{k}}{N} \\
\vec{C}_{i}=\overrightarrow{F c}_{i}-\vec{P}_{i}
\end{gathered}
$$

where $\overrightarrow{P_{k}}$ is the position of flight $k$ and $N$ is the number of neighbours. To compute this vector, neighbours flight are those located in the blue area of figure 3 .

If we apply only cohesion, separation and alignment rules, the aircraft will follow the same direction. Thus, we used a force that made each plane reach its specific destination. Actually, we are obliged to add a Destination Vector that steers each aircraft to its destination. But, adding only this force does not solve the whole problem. We notice that the trajectory of the aircraft becomes widely sinuous. This behavior is expected since boids are flexible in their movements, which is not allowed for airplanes trajectories. So, we add another force that represents the Previous Motion Vector. Hence, we introduce here two additional forces that we should be considered in our study.

d) Destination vector: this vector is introduced in order to oblige the flight to navigate toward its destination point. Thus, it is a vector that links the actual position of the flight with the destination position. It is denominated with $\vec{D}_{i}$ where $i$ is the flight index.

e) Previous motion vector: labelled $\overrightarrow{U_{\text {pred }}} \overrightarrow{\text {, is added in }}$ order to avoid oscillation of the trajectory. It corresponds to the displacement vector applied in the previous point 
of the trajectory. Obviously, we do not add this vector in the first point of the flight path.

Finally, the resulting vector, applied in each point of the $i^{\text {th }}$ flight path, is calculated as the weighted sum of the afore-mentioned five vectors as depicted in (9).

$$
\overrightarrow{U_{i}}=w_{1} * U_{p r e d}+w_{2} * \vec{D}_{i}+w_{3} * \vec{A}_{i}+w_{4} * \vec{S}_{i}+w_{5} * \vec{C}_{i}
$$

where $i$ is the flight index, and $w_{j} s$ are the coefficients used to balance the five forces describing the influence of each steering force.

4) Optimization process: The balance between different forces allows us to obtain better results. In our paper, for each aircraft, we intend to find the shortest path between departure and destination points while avoiding conflicts. The decision variables are the coefficients $w_{i}$, where $i$ varies from 1 to 5 , and the objective function is calculated as in (10).

$$
F_{o b j}=\operatorname{distance}\left(P_{f}, D_{f}\right)
$$

where $P_{f}$ represents the flight actual position, $D_{f}$ stands for its destination, and distance $\left(p_{1}, p_{2}\right)$ is the distance between two positions $p_{1}$ and $p_{2}$. Our goal is to minimize the objective function $F_{o b j}$ while eliminating all the conflicts. To achieve our aim, we propose to apply the simulated annealing algorithm. This optimization method is a methaheuristic algorithm inspired from thermodynamics theory where atoms, that have not yet acquired a globally optimal state of energy, move further until they reach stability. The idea of the simulated annealing is to accept changes even to a worse solution, relative to the current one, but in a controlled manner. In fact, the worse the neighbour is, the lower the probability of acceptance will be. This probability is called the temperature. A neighbourhood solution is generated by slightly modifying the coefficients $w_{i}$ from the current values. Therefore, for each coefficient we add or subtract a random value less inferior to 0.1. The whole process is split into two steps. The first stage is the heating up by increasing the temperature, which enhances the probability of acceptance in order to promote the exploration of the search space. Then, the cooling down schedule is launched by decreasing the temperature gradually in order to converge the system to a better solution. In our case, we diminish the temperature by applying a geometrical law.

First, we evaluate the number of conflict induced by the considered flight, $\Phi_{f 1}$, using 1 . Then, a neighbourhood solution is generated. After that, we calculate the new number of conflicts $\Phi_{f 2}$. If the new solution yields to a minimum number of conflicts, that is $\Phi_{f 1}>\Phi_{f 2}$, then it is accepted. In the case when the neighbourhood solution does not affect the number of conflicts, it will be accepted if it improves the objective function described in 10. Otherwise, the solution is accepted with the probability $e^{\left(\Phi_{f 1}-\Phi_{f 2}\right) / T}$ where $\mathrm{T}$ is the temperature (the simulated annealing parameter). After a predefined number of iterations, the temperature $\mathrm{T}$ is decreased by applying a geometrical law where the ratio is experimentally tuned.

\section{Results}

First of all, we have checked the efficiency of our algorithm with a small set of strategic aircraft paths leading to a considerable number of conflicts. The considered set contained 200 flights and was extracted from real eastbound traffic in 20 July 2012. It generated initially 3620 conflicts. We consider the separation standard as it is described in the former section, and we assume that the departure delay varies up to 20 minutes. Figure 4 displays 2D flight trajectories of the considered set before and after the application of our algorithm. Conflicts are shown via the red color in figure 4 . It is obvious that after applying our algorithm the number of conflict became significantly reduced to 39 conflicts. We note that 11 flights were delayed, and the path-elongation rate did not exceed $10 \%$. The extension path defined the rate of the extra path added to the direct route. To determine this rate, we calculated first the length of the direct route between entry and exit points denoted $d 1$. Then, we computed $d 2$, the sum of distances between each two consecutive points generated for each trajectory. The extension path is obtained as follows: $\frac{|d 1-d 2|}{d 1} * 100$.

Besides, the analysis was also conducted for four different sets of aircraft routes in July 2012. The number of flights in each set ranged from 368 to 649 . The number of conflicts produced by the different sets before and after the application of the algorithm is presented in I. The number of conflicts before and after the de-confliction method varied between [2321 - 4533] and [18 - 59], respectively. Even though a conflict-free solution did not exist, a significant reduction of the number of conflict was noticed.

As seen in table II, most of flights were not delayed. This result is reasonable since we used the alternative of delaying flights only for eliminating conflicts in their departure points. Obviously, it is recommended to be as closest as possible to the aircraft desired entry times in order to satisfy the preference of airline companies.

Table III represents statisticson of the rate of extension path regarding the direct one. The first important conclusion that can be deduced based on these results is that $75 \%$ of flights were within $1,2 \%$ from their direct routes. Moreover, about $50 \%$ of flights in each set took almost their direct routes. Thus, it is worth mentioning that fuel consumption was not affected by conflict reduction strategy since we had small variation from direct path between entry and exit points. However, one or two flights in each set are affected. Their extension paths exceed $10 \%$. In fact, the maximum of extension trajectory reached $17,6 \%$ for set 4 and $13,8 \%$ for set 1 . It affected respectively one and two flights. 


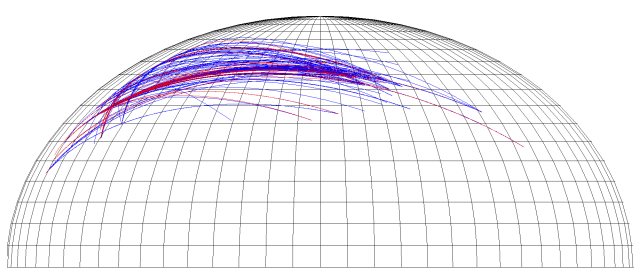

(a) Paths before the resolution algorithm

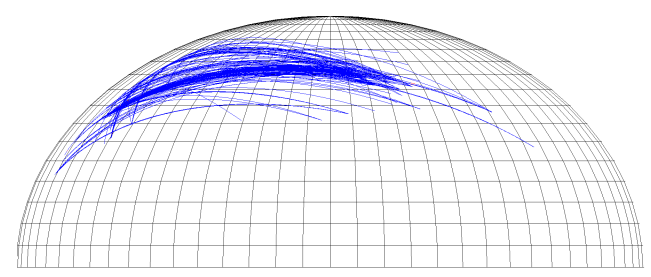

(b) Paths after the resolution algorithm

Fig. 4: A portion of real eastbound traffic in 20 July 2012

\begin{tabular}{|c|c|c|c|}
\hline Flight set & number of flights & Conflicts before & Conflicts after \\
\hline Set 1 & 368 & 2321 & 41 \\
\hline Set 2 & 617 & 2312 & 59 \\
\hline Set 3 & 572 & 3695 & 47 \\
\hline Set 4 & 649 & 4533 & 18 \\
\hline
\end{tabular}

TABLE I: Results of applying our approach for different sets of flights

\begin{tabular}{|c|c|c|c|}
\hline Test (number of flights) & \multicolumn{3}{|c|}{ number of flights } \\
\cline { 2 - 4 } & without delay & $0<$ delay $<=10 \mathrm{~min}$ & $10 \mathrm{~min}<$ delay $<=20 \mathrm{~min}$ \\
\hline Set 1 (368) & 366 & 2 & 0 \\
\hline Set 2 $(617)$ & 604 & 13 & 0 \\
\hline Set 3 $(572)$ & 565 & 6 & 1 \\
\hline Set 4 (649) & 642 & 6 & 1 \\
\hline
\end{tabular}

TABLE II: Delays for different flight sets

\begin{tabular}{|c|c|c|c|c|}
\hline Test (number of flights) & Set 1 (368) & Set 2 (617) & Set 3 (572) & Set 4 (649) \\
\hline First quartile & $0 \%$ & $0 \%$ & $0 \%$ & $0 \%$ \\
\hline Second quartile & $0 \%$ & $0 \%$ & $0 \%$ & $0,18 \%$ \\
\hline Third quartile & $0,009 \%$ & $0,05 \%$ & $0,005 \%$ & $1,18 \%$ \\
\hline Maximum extension & $13,8 \%$ & $10 \%$ & $13,09 \%$ & $17,6 \%$ \\
\hline
\end{tabular}

TABLE III: Evaluation of the distribution of extension path in comparison to direct route

\section{CONCLUSion}

This paper presents an efficient planning method for aircraft trajectories in the oceanic airspace. Assuming that all aircraft are equipped with the ADS-B system, we intended to improve the oceanic air traffic situation by reducing the separation standards. Based on the Flocking boid model, an algorithm for improving aircraft routes and avoiding conflicts has been implemented. In order to optimize the trajectory length, a method, based on simulated annealing algorithm has been applied. The algorithm has been tested with a real traffic data during July 2012. The computational results show that we can reduce considerably conflicts with reasonable delay and elongation from the direct path. Due to the highly-complex computations, we have been incapable to manage a bigger number of flights as it is the case for the current traffic that carries up to 1000 flights per day. The methodology adopted in this paper has been programmed sequentially. In the future work, we look forward to better the aforementioned results with a parallel computing program.

\section{REFERENCES}

[1] NAT DOC 007. Guidance concerning air navigation in and above the north atlantic mnps airspace. Edi- tion 2010. World Air Ops online Library.

[2] Craig W. Reynolds. Flocks, herds and schools: A distributed behavioral model. volume 21, pages 2534, New York, NY, USA, August 1987. ACM.

[3] I.R. de Oliveira, F.S. Carvalho, J. Batista Camargo, and L.M. Sato. Multi-agent tools for air traffic management. In Computational Science and Engineering Workshops, 2008. CSEWORKSHOPS '08. 11th IEEE International Conference on, pages 355-360, July 2008.

[4] H. Emami and F. Derakhshan. An overview on conflict detection and resolution methods in air traffic management using multi agent systems. In Artificial Intelligence and Signal Processing (AISP), 2012 16th CSI International Symposium on, pages 293-298, May 2012.

[5] A. Parkinson. Space-based ads-b: A small step for technology a giant leap for atm? In Digital Communications - Enhanced Surveillance of Aircraft and Vehicles (TIWDC/ESAV), 2011 Tyrrhenian International Workshop on, pages 159-164, Sept 2011.

[6] H. Blomenhofer, P. Rosenthal, A. Pawlitzki, and L. Escudero. Space-based automatic dependent 
surveillance broadcast (ads-b) payload for in-orbit demonstration. In Advanced Satellite Multimedia Systems Conference (ASMS) and 12th Signal Processing for Space Communications Workshop (SPSC), 2012 6th, pages 160-165, Sept 2012.

[7] Shon Grabbe, Banavar Sridhar, and Nadia Cheng. Central east pacific flight routing. Air Traffic Control Quarterly, 15(3):239-264, 2007.

[8] Shon Grabbe, Banavar Sridhar, and Avijit Mukherjee. Central east pacific flight scheduling. In $A I A A$ Guidance, Navigation, and Control Conference and Exhibition, Hilton Head, South Carolina, 2007.

[9] Olga Rodionova, Mohammed Sbihi, Daniel Delahaye, and Marcel Mongeau. Optimization of aicraft trajectories in north atlantic oceanic airspace. In ICRAT 2012, 5th International Conference on Research in Air Transportation, pages pp-xxxx, 2012.

[10] Banavar Sridhar, Neil Y Chen, K Ng Hok, Olga Rodionova, Daniel Delahaye, and Florian Linke. Strategic Planning of Efficient Oceanic Flights. In ATM seminar 2015, 11th USA/EUROPE Air Traffic Management R\&D Seminar, Lisboa, Portugal, June 2015. FAA \& Eurocontrol. 